\title{
Study of ferroelectric liquid crystal modulators for their use in diffractive and polarization optical applications
}

\section{Estudio de moduladores de cristal líquido ferroeléctrico para su uso en aplicaciones de óptica difractiva y de polarización}

\author{
Antonio Martínez García $\left.{ }^{*}, \mathrm{~S}\right)$ \\ Departamento de Ciencia de Materiales Óptica y Tecnología Electrónica, Universidad Miguel Hernández de \\ Elche, Avda. Universidad s/n, 03202, Elche, Alicante, Spain. \\ (*) Email: catovillena@gmail.com \\ S: miembro de SEDOPTICA / SEDOPTICA member \\ Received / Recibido: 24/10/2014. Revised / Revisado: 11/11/2014. Accepted / Aceptado: 12/11/2014. \\ DOI: http://dx.doi.org/10.7149/OPA.47.4.273
}

\begin{abstract}
:
This paper presents a summary of the $\mathrm{PhD}$ thesis with the same name. Specifically, the following issues are addressed: 1) Development of a calibration method of the physical parameters for ferroelectric liquid crystal modulators, 2) use of ferroelectric liquid crystal modulators in diffractive optics and polarization, and 3) use of multiple characterization techniques to assess the quality of the computer generated holograms to be fabricated on silicon wafers.
\end{abstract}

Key words: Liquid Crystal modulators, Phase and Amplitude Optical Modulation, Diffraction, Polarization.

\section{RESUMEN:}

Este trabajo presenta el resumen de la tesis doctoral con el mismo nombre. En concreto, se tratan los siguientes aspectos: 1) Desarrollo de un método de calibración de los parámetros físicos para moduladores de cristal líquido ferroeléctrico, 2) empleo de moduladores de cristal líquido ferroeléctrico en óptica difractiva y de polarización, y 3) empleo de múltiples técnicas de caracterización evaluar la calidad de hologramas generados por ordenador que se fabricaron sobre obleas de silicio.

Palabras clave: Moduladores de Cristal Líquido, Modulación Óptica de Fase y Amplitud, Difracción, Polarización.

\section{REFERENCES AND LINKS / REFERENCIAS Y ENLACES}

[1]. A. Martínez García, Estudio de Moduladores de Cristal Líquido Ferroeléctrico para su Uso en Aplicaciones de Óptica Difractiva y de Polarización, PhD Thesis, Universidad Miguel Hernández de Elche (2011).

[2]. L. Sirleto, G. Coppola, G. Breglio, G. Abbate, G.C. Righini, J. M. Otón, "Electro-optical switch and continuously tunable filter based on a Bragg grating in a planar waveguide with a liquid crystal overlayer", Opt. Eng. 41, 2890-2898 (2002). DOI

[3]. A. Martínez, N. Beaudoin, I. Moreno, M. M. Sánchez-López and P. Velásquez, "Optimization of the contrast-ratio of a ferroelectric liquid crystal optical modulator", J. Opt. A: Pure App. Opt. 8, 1013-1018 (2006). DOI

[4]. C. Maurer, A. Schwaighofer, A. Jesacher, S. Bernet, M. Ristch-Marte, "Suppression of undesired diffraction orders of binary phase holograms", Appl. Opt. 47, 3994-3998 (2008). DOI

[5]. A. Jaulin, L. Bigué, "High speed partial Stokes imaging using a ferroelectric liquid crystal modulator", J. Eur. Opt. Soc. Rapid Pub. 3, 08019 (2008). DOI 
[6]. G. Moddel, "Ferroelectric liquid crystal spatial light modulators", Chapter 6 in Spatial Light Modulator Technology, U. Efron, Edt. pp. 287-359, Marcel Dekker Inc., New York (1995).

[7]. I. Underwood, "Ferroelectric liquid crystal over silicon spatial light modulators- principles, practice and prospects", in OSA Trends in Optics and Photonics vol. 14, Spatial Light Modulators, G. Burge and S. C. Esener Edts., pp. 76-88 (1997).

[8]. X. H. Li, A. Murauski, A. Muravsky, P. Z. Xu, H. L. Cheung, V. G. Chigrinov, "Grayscale generation and stabilization in ferroelectric liquid crystal display", J. Disp. Tech. 3, 273-279 (2007). DOI

[9]. P. Hariharan, P. E. Ciddor, "Improved switchable achromatic phase shifters", Opt. Eng. 38, 10781080 (1999). DOI

[10]. D. J. McKnight, D. G. Vass, R. M. Sillitto, "Development of a spatial light modulator: a randomly addressed liquid-crystal-over-nMOS array", Appl. Opt. 28, 4757-4762 (1989). DOI

[11]. N. Collings, W. A. Crossland, P. J. Ayliffe, D. G. Vass, I. Underwood, "Evolutionary development of advanced liquid cristal spatial light modulators", Appl. Opt. 28, 4740-4747 (1989). DOI

[12]. K. Heggarty, B. Fracasso, C. Letort, J. L. de Bougrenet de la Tocnaye, M. Brich, D. Krüerke, "Silicon blackplane ferroelectric liquid crystal spatial light modulator for uses within an optical telecommunications environment", Ferroelectrics 312, 39-55 (2004).

[13].J. L. Martínez, A. Martínez-García, I. Moreno, "Wavelength compensated color Fourier diffractive optical elements using a ferroelectric liquid crystal on silicon display and a color-filter wheel", Appl. Opt. 48, 911-918 (2009). DOI

[14]. http://www.forthdd.com/products (last visited in 21/11/2014)

[15]. P. Velásquez, M. M. Sánchez-López, I. Moreno, D. Puerto, F. Mateos, "Interferente birefringent filters fabricated with low cost commercial polymers", Am. J. Phys. 73, 357-361 (2005). DOI

[16]. A. Martínez-García, I. Moreno, M. M. Sanchez-Lopez, P. García-Martínez, "Operational modes of a ferroelectric LCoS modulator for displaying binary polarization, amplitude, and phase diffraction gratings", Appl. Opt. 48, 2903-2914 (2009). DOI

[17]. M. M. Sánchez-López, P. García-Martínez, A. Martínez-García, I. Moreno, "Poincaré sphere analysis of a ferroelectric liquid crystal optical modulator: application to optimize the contrast ratio", J. Opt. A: Pure Appl. Opt. 11, 015507 (2009). DOI

[18].A. Martínez, M. M. Sánchez-López, I. Moreno, "Phasor analysis of binary amplitude gratings with different fill factor", Eur. J. Phys. 28, 805-816 (2007). DOI

[19]. I. Moreno, M. J. Yzuel, J. Campos y A. Vargas, "Jones matrix treatment for polarization Fourier optics", J. Mod. Opt. 51, 2031-2038 (2004). DOI

[20]. A. Martínez, I. Moreno, M. M. Sánchez-López, "Comparative analysis of time and spatial multiplexed diffractive optical elements in a ferroelectric liquid crystal", Jap. J. Appl. Phys. 47, 1589-1594 (2008). DOI

[21]. I. Moreno, C. Iemmi, A. Márquez, J. Campos, M. J. Yzuel, "Modulation light efficiency of diffractive lenses displayed in a restricted phase-mostly modulation display", Appl. Opt. 43, 6278-6284 (2004). DOI

[22]. I. Moreno, A. Martínez-García, L. Nieradko, J. Albero, C. Gorecki, "Low cost production of computergenerated holograms: from design to optical evaluation", J. Eur. Opt. Soc.-Rapid Publications 5, 10011 (2010). DOI

[23]. A. Martínez-García, I. Moreno, P. Velásquez, A. Salinas, R. Mallavia, "Elipsometría espectroscopía de ángulo variable: una técnica para la determinación precisa de constantes ópticas y espesores", Rev. Esp. Fis. 25, (2011).

\section{Introducción}

El contenido de este artículo es un breve resumen de los principales resultados obtenidos en la elaboración de la tesis titulada "Estudio de moduladores de cristal líquido ferroeléctrico para su uso en aplicaciones de óptica difractiva y de polarización" [1]. El trabajo se centró principalmente en el estudio de dos moduladores ópticos de cristal líquido ferroeléctrico (FLC), concretamente en la calibración de sus parámetros físicos, sus modos de operación en modulación óptica y su empleo para la realización de elementos ópticos difractivos y de polarización.

Los moduladores FLC actúan en modo binario, correspondiente a las dos posiciones estables del director molecular del cristal líquido, por lo que son dispositivos útiles para la 
realización de conmutadores ópticos, y de SLM que realicen elementos binarios. La capa de cristal líquido es de fase esméctica $\mathrm{Sm}-\mathrm{C}^{*}$, que presentan una estructura molecular estratificadas en capas, con un cierto ángulo de inclinación respecto de los distintos planos de estas capas, y describen una rotación alrededor del eje que forma el vector normal a los planos. $\mathrm{Si}$ el material se confina entre dos placas separadas por una distancia muy pequeña, inferior al periodo de la estructura helicoidal, se adoptala estructura conocida de cristal líquido ferroeléctrico estabilizado en superficie (SSFLC, del inglés Surface Stabiliced Ferroelectric Liquid Crystal] [2]. Este confinamiento hace que el vector de polarización eléctrica espontánea solamente puede adoptar dos posiciones, siempre perpendicular al plano del substrato, y con los dos posibles sentidos opuestos. La aplicación de este campo eléctrico sobre la estructura SSFLC provoca un giro del director molecular (Fig. 1), que se reorienta con un ángulo $\pm \Delta \theta / 2$ en el plano del substrato (perpendicular al campo eléctrico). Cuando cambia el signo del campo eléctrico aplicado, las moléculas se reorientan con un ángulo opuesto $[2,3]$. Por este motivo los moduladores FLC se alimentan con señales de voltaje simétricas de valor medio nulo, obteniéndose así dos estados simétricos (dependiendo del nivel de señal eléctrica empleada). El valor medio nulo asegura un tiempo de vida largo al dispositivo ya que de otra manera, una componente DC del campo eléctrico puede degradar la fase del cristal líquido. El giro neto de las moléculas es por tanto el doble del ángulo de inclinación $(\Delta \theta=2 \theta)$.

Así pues, los moduladores SSFLC pueden considerarse como una lámina birrefringente uniáxica, con su eje óptico situado en el plano de la lámina y definido por la orientación del director del FLC, que puede conmutar entre dos estados estables, con un ángulo relativo $\Delta \theta$, mediante la aplicación de un voltaje. Los moduladores ópticos FLC son particularmente interesantes dada su alta velocidad de conmutación en comparación con los moduladores de cristal líquido nemáticos, que tienen un tiempo de respuesta lento, del orden de los milisegundos. Los moduladores FLC, por el contrario, son capaces de operar a varios $\mathrm{kHz}$ $[4,5]$ y presentan una alta eficiencia eléctrica por su bajo consumo [6,7]. Aunque se trata de dispositivos binarios, que solo poseen dos posiciones estables del director molecular, pueden aplicarse técnicas de multiplexado temporal para producir niveles de irradiancia intermedios [8], lo que los hace útiles también como displays de visualización de imágenes. Los moduladores FLC monopíxel se han utilizado como obturadores ópticos y elementos para óptica adaptativa [2], o como rotores ópticos de polarización y desfasadores conmutables [9].

A finales de la década de los 80 se fabrican los primeros moduladores de cristal líquido ferroeléctrico sobre silicio, o modulador FLCoS $[10,11]$. Los displays pixelados FLCoS se vienen utilizando como micropantallas de visión directa, y en sistemas de proyección. Además, se han empleado en otro tipo de aplicaciones, desde los sistemas de polarimetría [5], sistemas de comunicación [12] o en óptica difractiva [13]. El tamaño del área del píxel varía según el fabricante, oscilando entre unas decenas de micra hasta tamaños inferiores a las $8 \mathrm{~mm}$ [4]. La disminución en el tamaño del píxel que se ha obtenido con la tecnología LCoS ha facilitado aumentar espectacularmente la resolución y eficiencia, pasando de las primeras matrices de $50 \times 50$ píxeles a las actuales que superan normalmente los $1000 \times 1000$ píxeles [14].

Los moduladores que se emplearon en la Tesis fueron dos, ambos de la marca CRL-Opto, fueron un modulador monopíxel en transmisión, modelo LCS2-G, y un modulador pixelado de

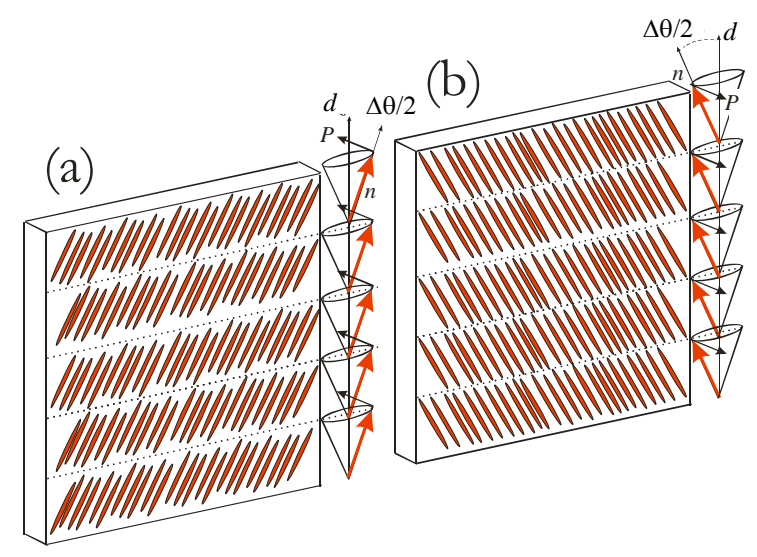

Fig. 1. Representación de los dos estados estables en una estructura SSFLC: $d$ es la normal a las capas esmécticas, $n$ es el director molecular del FLC, $\Delta \theta$ es el ángulo neto de giro de las moléculas y $P$ es la polarización eléctrica espontánea. 
tecnología FLCoS, modelo RXGA 1.5C. El dispositivo monopíxel se empleó para desarrollar un método de caracterización de los principales parámetros físicos, y para estudiar las configuraciones de modulación óptica.

Con el dispositivo pixelado se comprobó la validez del método de caracterización, y se empleó para realizar elementos de óptica difractiva y de polarización. Los elementos de óptica difractiva realizados en este dispositivo se sometieron a un estudio de la eficiencia de difracción.

Finalmente, se presentó un estudio experimental sobre la aplicación de técnicas ópticas de caracterización para el análisis de propiedades de elementos hologramas generados por ordenador y fabricados mediante técnicas de microfabricación.

\section{Análisis del modo de operación del modulador FLC monopíxel}

El modulador monopíxel se empleó para desarrollar un procedimiento sistemático de ingeniería inversa que caracteriza, a partir de la respuesta óptica del modulador FLC, sus parámetros físicos tales como la orientación del director molecular, el desfase $\varphi$, y la rotación molecular $\Delta \theta$ que se produce cuando se aplica un campo eléctrico bipolar.

En este modulador monopíxel comprobamos por vez primera la técnica propuesta para elementos pasivos [15], extendiéndola para la obtención experimental de los parámetros físicos de los moduladores FLC [3]. Determinamos, mediante la aplicación de una señal de voltaje bipolar de media nula, la orientación del director molecular en cada uno de los dos estados estables, obteniendo asimismo el valor de desfase para varias longitudes de onda. Comprobamos la dispersión cromática del valor del desfase al variar la longitud de onda, y propusimos una alternativa a la medida inicial cuando el desfase se hace muy próximo a $\varphi=180^{\circ}$, situación en la que el error en la precisión de la medida diverge.

Este método de ingeniería inversa se empleó también en la caracterización de los parámetros físicos del modulador pixelado FLCoS [16]. Por tanto, concluimos que este método resulta muy útil cuando los fabricantes no proporcionan los parámetros necesarios para obtener un buen rendimiento de los dispositivos. Se ha planteado el modo de operación ideal como modulador de irradiancia, situación que se obtiene para un desfase de $\varphi=180^{\circ}$ y un rotación del director molecular de $\Delta \theta=45^{\circ}$. Entonces, el FLC actúa como un desfasador de media onda reorientable $y$, si se emplea una iluminación linealmente polarizada, los dos estados de polarización emergentes del modulador son linealmente polarizados y ortogonales entre sí. Por tanto, se obtiene un contraste máximo en la irradiancia óptica si se orienta el analizador perpendicular a cualquiera de estos dos estados. Esta situación se ilustra en la Fig. 2.

Cuando el desfase $\varphi$ caracterizado es distinto a $180^{\circ}$ la respuesta en irradiancia del modulador FLC sufre una merma en el contraste óptico. Para compensar esta reducción se propuso un método que introduce un desfasador de cuarto de onda entre el polarizador de entrada y el modulador FLC [3]. Este sistema genera una polarización elíptica en el haz de entrada al modulador, que se puede seleccionar de modo que los dos estados emergentes del modulador FLC sean lo más próximos posible a ser lineales y ortogonales entre sí, con lo que se obtiene una optimización del contraste de la señal de
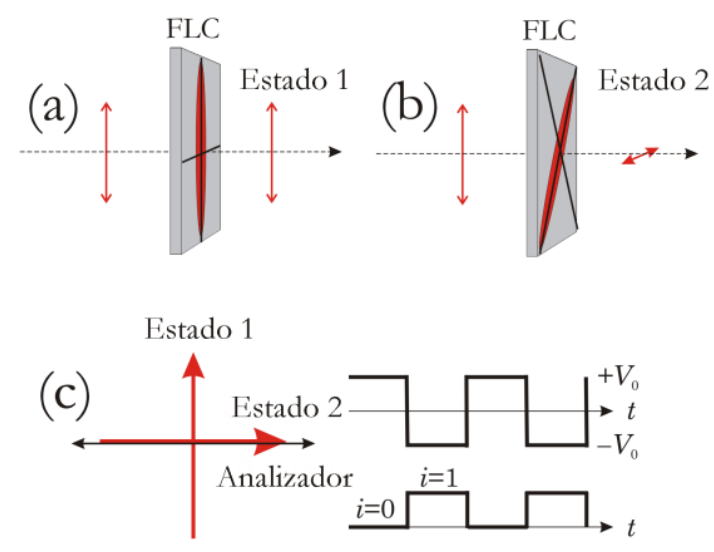

Fig. 2. Modo estándar de operación de un modulador FLC ideal (desfase $\varphi=180^{\circ}$ y rotación $\Delta \theta=45^{\circ}$ ) como modulador de irradiancia. La polarización de entrada es lineal y paralela al director en el estado 1. La orientación del analizador se indica con la flecha negra en (c). (a) Salida en estado 1. (b) Salida en el estado 2. (c) Configuración de polarización para obtener modulación binaria de irradiancia $i(t)$ en función de un voltaje binario $V(t)$. 
irradiancia óptica. El desfasador puede también introducirse entre el modulador FLC y el analizador y obtener los mismos resultados de optimización. Se analizó este método de optimización visualizando las transformaciones del estado de polarización sobre la esfera de Poincaré, lo que nos permitió comprobar que el problema de localizar los ángulos de los polarizadores y del desfasador se reduce a la resolución de un problema de geometría esférica. Empleando las relaciones de trigonometría esférica se obtuvo las expresiones analíticas del contraste en función del valor de desfase $\varphi$, tanto para la configuración estándar como para la configuración optimizada [17].

Verificamos experimentalmente las ideas propuestas con el modulador monopíxel FLC, empleando dos láseres de He-Ne diferentes, con longitudes de onda $543 \mathrm{~nm}$ y $633 \mathrm{~nm}$ respectivamente. El desfase inducido para la primera longitud de onda, muy cercano a $180^{\circ}$, permitió operar el modulador FLC de forma ideal. Para el segundo caso, el desfase era solamente de $135^{\circ}$ y, por tanto, se empleó el método de optimización propuesto para mejorar su respuesta óptica. Se obtuvo un excelente resultado en la mejora del contraste de la irradiancia óptica.

\section{Aplicación de un dispositivo FLCoS en óptica difractiva}

El modulador pixelado con tecnología FLCoS se empleó para la realización de elementos de óptica difractiva, en particular redes de difracción, axicones, lentes generadoras de vórtices, y hologramas generados por ordenador. Tal y como se ha mencionado, previamente a su uso aplicamos la técnica de ingeniería inversa propuesta para el modulador monopíxel y así obtener los parámetros físicos (orientación del director molecular y desfase) de este otro modulador. Como resultado de la calibración, se pudo determinar las orientaciones de los polarizadores para operar el dispositivo en modo de modulador de amplitud o modulador de fase [13]. En ambos casos, el display opera de modo binario, por lo que debimos restringirnos a la realización de elementos difractivos binarios. También se comprobó el control electrónico del display, que muestra una secuencia temporal de las componentes RGB de la imagen en color que se visualiza en el modulador [16].

El primer elemento difractivo que se realizó en el modulador fueron redes de difracción [16]. En primer lugar se analizó la realización de redes binarias de amplitud con dicho dispositivo. Se presentó una descripción completa de estas redes $y$, en particular, del efecto de variar el factor de llenado (relación entre las anchuras de los dos estados de la red). Analizamos este problema en base a la teoría de la óptica de Fourier, pero también desarrollamos una técnica fasorial [18] que describe fácilmente el patrón de difracción sin necesidad de acudir al tratamiento por transformada de Fourier. Posteriormente, se extendió la técnica fasorial también al análisis de redes binarias de fase. La propuesta de la técnica fasorial es físicamente muy intuitiva y permite calcular la irradiancia de los órdenes de difracción. Por tanto, este tratamiento provee de una potente herramienta, útil para la comprensión intuitiva así como para la enseñanza de estos aspectos en cursos de Física general, en los que la difracción no puede ser aún expuesta mediante la teoría de la óptica de Fourier.

Los resultados experimentales obtenidos con el display FLCoS operando como modulador binario de amplitud verificaron las predicciones teóricas, y constituyen una buena demostración óptica para ilustrar las redes de difracción.

A continuación, se presentó un estudio completo de redes de difracción de polarización (PDG) binarias realizadas mediante el display FLCoS. Aplicamos el formalismo de JonesFourier [19] para analizar una PDG binaria constituida por dos regiones formadas por desfasadores que introducen un desfase arbitrario $\varphi$ con una rotación de sus ejes neutros de $\Delta \theta=45^{\circ}$ (se consideraron orientados en $0^{0}$ y $45^{\circ}$ respectivamente) [16]. El estudio reveló que, al igual que ocurre en las redes binaria escalares, la red PDG binaria con un factor de llenado del $50 \%$ produce un patrón sin órdenes pares. La acción de la PDG y la propagación hasta el plano de Fourier puede ser equivalente a la de un desfasador de media onda orientado a $-22,5^{\circ}$ para los órdenes difractados 
impares, con independencia del desfase $\varphi$. Por el contrario, la acción sobre el orden cero produce una polarización elíptica. No obstante, cuando el desfase es de $\varphi=180^{\circ}$, la acción en el orden cero es equivalente a la de un desfasador de media onda orientado a $+22,5^{\circ}$.

Basados en este análisis de polarización, se mostró cómo estas redes PDG son una generalización de las redes de amplitud o de fase, que se obtienen mediante la conveniente proyección de los estados de polarización sobre un analizador. Como resultado de este análisis presentamos dos mejoras en el sistema óptico que emplea el display FLCoS para la generación de elementos difractivos. La primera consiste en una simplificación que emplea un único polarizador, que actúa tanto sobre el haz incidente como sobre el reflejado. Esta simplificación genera resultados de modulación escalar (amplitud o fase) equivalentes a emplear dos polarizadores. En segundo lugar analizamos la respuesta de la PDG constituida por desfasadores con desfase $\varphi \neq 180^{\circ}$. Comprobamos que se produce una reducción de la eficiencia de difracción. Sin embargo, demostramos que se puede obtener una respuesta casi equivalente a la de la red de amplitud o la de fase iluminando el modulador con una polarización elíptica. Obtuvimos una respuesta en difracción característica de las redes binarias de fase $\pi$, con desfases muy alejados del valor ideal $\varphi=180^{\circ}$, mejorando sensiblemente la eficiencia relativa de difracción para estas longitudes de onda.

Estos desarrollos teóricos fueron experimentalmente comprobados con dos láseres de diferente longitud de onda: una (568 nm) hace que el modulador actúe como desfasador de media onda, mientras que la otra (647 nm) muestra un desfase muy alejado al ideal. Este estudio es, por tanto, una guía de uso para la realización de redes PDG, redes de amplitud o redes de fase con el modulador FLCoS, lo que es interesante para aplicaciones de óptica difractiva programable que empleen estos dispositivos.

A continuación, se realizó un estudio comparativo de técnicas de multiplexado de elementos de difracción [20]. Se presentaron dos técnicas para la realización de un multiplexado temporal de elementos ópticos difractivos. El primer método aprovecha el funcionamiento electrónico de nuestro dispositivo FLCoS, diseñado para la visualización de imágenes a color, que hace que las componentes RGB sean secuencialmente visualizadas en el modulador. En nuestro caso, al iluminar con una fuente láser continua monocromática se pudo directamente multiplexar temporalmente tres elementos difractivos simplemente añadiendo cada uno en cada uno de los canales RGB de la imagen que controla el modulador. Se compararon los resultados obtenidos con esta técnica temporal con varias técnicas de multiplexado espacial propuestas en la literatura, para las cuales se analizó teórica y numéricamente su degradación respecto de la respuesta ideal. Los resultados para la técnica temporal mostraron claramente su ventaja frente a las otras técnicas cuando se emplean detectores capaces de integrar temporalmente la secuencia completa. Mostramos esta mejora tanto en simulaciones por ordenador como en los resultados experimentales obtenidos con el modulador FLCoS.

Del estudio de las configuraciones de modulación del dispositivo se derivó una configuración específica que genera modulación binaria de fase $\pi$ independientemente de la longitud de onda empleada [13]. La configuración es muy simple y no requiere desfasadores adicionales para compensar el efecto del desfase. El polarizador de entrada debe orientarse en la bisectriz de las dos orientaciones estables del director del FLC, mientras que el analizador se orienta perpendicular a éste. Empleamos el formalismo de Jones para analizar las propiedades de modulación óptica y caracterizar las propiedades de la eficiencia de difracción de esta configuración. Las variaciones de desfase afectan al nivel global de transmisión, que lógicamente afecta a la eficiencia de difracción or modulación [21]. Pero la actuación relativa con dos niveles con diferencia de fase $\pi$ permite cancelar el orden cero de difracción, independientemente del valor de desfase $\varphi$ del modulador (y por tanto, con independencia de la longitud de onda) lo que permite su utilización en aplicaciones con luz policromática. 
Obtuvimos resultados experimentales con redes de difracción, que verifican la cancelación del orden cero en un amplio rango de longitudes de onda. Asimismo aplicamos una técnica de multiplexado temporal para compensar el efecto de la dispersión cromática propia de la difracción, combinando la secuencia RGB de los elementos difractivos reescalados con la secuencia RGB de iluminación con tres líneas láser filtradas mediante una rueda de filtros de color [13]. Aprovechamos estas propiedades de modulación y la versatilidad del sistema para generar elementos difractivos con respuesta axial, tales como axicones o lentes para producir vórtices ópticos. En estos casos, la selección de la configuración de modulación binaria de fase $\pi$ para un amplio rango de longitudes de onda resulta imprescindible para evitar el solapamiento de la componente de luz no difractada correspondiente al orden cero.

En resumen, el trabajo desarrollado con los dos moduladores FLC supuso un estudio en profundidad en aspectos clásicos de la óptica como son la polarización y la difracción, especialmente por implicación en el modo de utilización de dispositivos electro-ópticos moduladores de cristal líquido ferroeléctrico. El trabajo desarrolló contenidos teóricos novedosos, en aspectos tales como el estudio de los modos de operación de los FLCDs, o el estudio de redes de polarización. Sin embargo, se debe destacar que fue un trabajo básicamente de laboratorio, incluyendo aspectos como la calibración de los dispositivos, y su empleo para la realización de diversos elementos ópticos.

\section{Inspección óptica de elementos difractivos fabricados sobre obleas de silicio}

Por último, la parte final de la Tesis estuvo dedicada a la aplicación de dos técnicas ópticas de inspección para evaluar la calidad de hologramas generados por ordenador (CGH, del inglés Computer Generated Holograms) que se fabricaron sobre obleas de silicio [22]. Esta parte final no tiene relación con los moduladores FLC, pero sí está relacionada con el diseño de elementos difractivos.
Se presentó un proceso completo de desarrollo de elementos CGH de bajo coste y con alta calidad. Este proceso incluyó:

1) La optimización del diseño basado en el algoritmo iterativo de transformadas de Fourier,

2) Su codificación directa como CGH de fase (kinoform) o como CGH de fases por desplazamiento,

3) La fabricación de los CGH sobre obleas de $\mathrm{SiO}_{2} /$ Si mediante la microfabricación,

4) La aplicación de la técnica de elipsometría espectral [23] para determinar el espesor de la capa de $\mathrm{SiO}_{2}$, y emplear este dato para calcular las reflexiones complejas en los dos niveles del holograma,

5) La inspección óptica de la fase relativa en función de la longitud de onda mediante microscopía interferencial, como una técnica simple para visualizar los rangos de longitudes de onda que proporcionan las mejores eficiencias de difracción,

6) El cálculo de la eficiencia de difracción y la evaluación experimental de la calidad de la reconstrucción de los hologramas.

La Fig. 3 ilustra uno de los pasos, en el cual se empleó un microscopio con un objetivo interferencial. Mediante el desplazamiento lateral de las franjas de interferencia se pudo determinar muy fácilmente de manera visual la
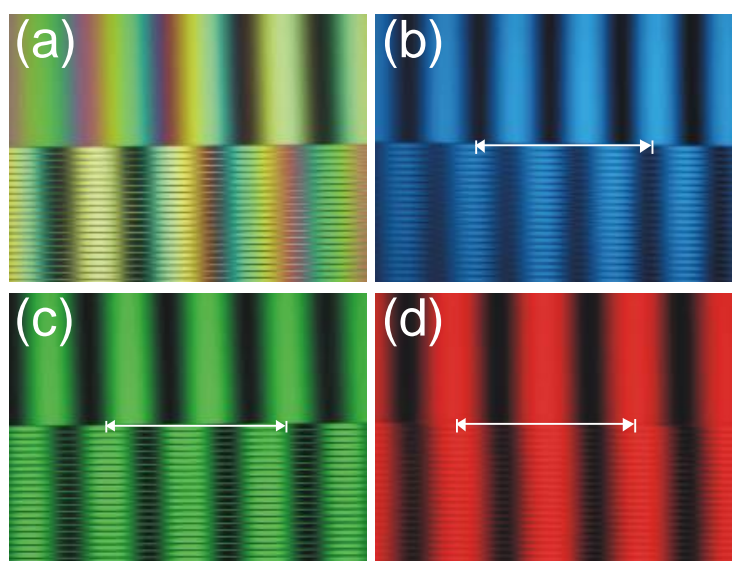

Fig. 3. Imágenes de la red de difracción capturadas con el microscopio con objetivo interferométrico diferencial 20x: (a) iluminación con luz blanca, (b) y con filtro en $488 \mathrm{~nm}$, (c) $543 \mathrm{~nm}, \mathrm{y}$ (d) $633 \mathrm{~nm}$ respectivamente [22] 
diferencia de fase entre las dos zonas grabadas en el holograma, en función de la longitud de onda. Mediante la incorporación de filtros interferenciales en el sistema de iluminación, pudimos seleccionar diferentes longitudes de onda a partir de la lámpara de luz blanca. Así, por ejemplo, en la Fig. 3(d) se observa que para luz roja la diferencia de fase es aproximadamente de $4 \pi$ radianes, por lo que se puede prever una muy baja eficiencia de difracción, tal y como posteriormente se comprobó.

La evaluación de los coeficientes complejos de reflexión en las dos zonas de los hologramas se reveló de gran importancia para la correcta evaluación de la eficiencia de difracción de los elementos fabricados, tanto en cuanto a las pérdidas introducidas por absorción, como por el importante efecto interferencial de lámina delgada.

El proceso expuesto en le ref. [22] representa una guía completa para la inspección del proceso de fabricación de CGH, y recibió el premio EOS2011 de la European Optical Society. La fabricación de los CGH sobre $\mathrm{SiO}_{2} / \mathrm{Si}$ se llevó a cabo en colaboración con investigadores del Institut FEMTO, de la Université de Franche-
Comté, Besançon (Francia), y muestra una excelente calidad en el patrón de reconstrucción. Sin embargo presentan unas eficiencias de difracción bajas debido a las fuertes pérdidas por reflexión. No obstante, los CGH fabricados son buenos candidatos para actuar como moldes en el replicado directo sobre polímeros o mediante microinyección.

\section{Conclusiones}

En definitiva, se han resumido las principales contribuciones teóricas y prácticas desarrolladas en la tesis mencionada [1], donde pudimos realizar un amplio estudio aplicado a moduladores de cristal líquido. Además la tesis tuvo un alto valor por la gran cantidad de técnicas de caracterización empleadas que van desde el uso de elementos ópticos pasivos hasta técnicas complejas como la microscopía interferencial o la elipsometría.

\section{Agradecimientos}

Agradezco el puesto de Técnico de investigación sujeto a un proyecto de investigación del Ministerio de Educación y Cultura de España (proyecto FIS2006-13037-C02-02), gracias al cual pude desarrollar el trabajo descrito. 